\title{
CITADELLE ASSIÉGÉE, CITADELLE ASSAILLIE, ARCHITECTURES ET ÉCRITURES DE LA RETRAITE DANS LE ROMAN SADIEN
}

Chez Kant, quand on a retiré tous les objets, il reste l'espace. Du moins est-ce ainsi que le professeur de philosophie explique parfois à ses élèves le statut des formes $a$ priori de la sensibilité dans la Critique de la raison pure. Il en va de même, si l'on peut dire, chez Sade (compagnon du premier, depuis Lacan). Dans le souvenir et l'imagination de son lecteur, une fois que se sont estompés les personnages de son œuvre, une fois leurs dissertations oubliées, une fois fondus et confondus les détails de leurs infamies, que reste-t-il sinon l'espace de la fiction ? Que reste-t-il, dans cette recomposition par le souvenir, sur cette cartographie souterraine de la mémoire du lecteur dont avait parlé Julien Gracq ${ }^{1}$, sinon, surtout, les sites et les demeures extraordinaires des libertins ? Leurs murs ont comme absorbé toute l'atmosphère de l'œuvre et ils suffisent presque à en restituer la radicalité. Si bien que l'œuvre se confond, dans notre imaginaire, avec ses propres architectures, tout comme le Sade de Man Ray dont le buste est pétri des pierres de sa prison. Car c'est bien la prison qui, parmi tous les espaces sadiens, sert de paradigme, dans un dégradé de biographie et de fiction qui va de Vincennes et la Bastille, au château de Silling et aux pavillons souterrains de Sainte-Marie-des-Bois. Cette sorte de reductio ad carcerem s'impose dès l'aube des études sadiennes. Elle informe tout aussi bien les puissantes rêveries d'un Gilbert Lely, qui, pour reconstituer le roman à jamais perdu des Journées de Florbelle, lui donnait l'allure d'un château à mi- chemin entre Silling et la Bastille ${ }^{2}$, que les travaux universitaires de Jean-Jacques Brochier, lequel repérait, dans un article fondateur, quatre caractéristiques de l'espace romanesque sadien : la solitude, le secret, le cellulaire, et la verticalité ${ }^{3}$. C'est dire si le roman sadien se donne sous les aspects d'une gigantesque retraite.

Reste à savoir de quel côté du mur se trouve le libertin. La question peut sembler absurde si l'on s'en tient aux romans « infâmes » de Sade (les Cent vingt journées de Sodome, les versions de Justine et l'Histoire de Juliette) où le scélérat, de Durcet à Minski, s'enterre avec ses victimes. Mais le motif de la retraite fortifiée prend des sens et des formes différents si l'on considère l'œuvre avouée et signée de Sade (Aline et Valcour, les Crimes de l'amour ou encore les romans historiques dont La marquise de Gange) - où ce motif occupe également une grande place, pourtant beaucoup moins

\footnotetext{
${ }^{1}$ «Le souvenir qu'on garde d'une œuvre de fiction de longue haleine, d'un roman, lu ou relu pour la dernière fois il y a des années, après tout le travail de simplification, de recomposition, de fusion, de rééquilibrage qu'entraine l'élision de la mémoire, fournirait, si la matière n'en était par nature aussi évasive, un sujet d'étude bien intéressant [...] Un autre livre apparaîtrait sous le premier - comme un autre tableau apparaît sous le tableau radiographié - qui serait un peu ce qu'est à la carte économique d'un pays celle de ses seules sources d'énergie ». (Julien Gracq, En lisant, en écrivant, Paris, José Corti, 1980, p. 130).

${ }^{2}$ «Les murailles écarlates des récits de Modose et d'Emilie; les quatre tours d'angle des dissertations (...); les huit tours flanquantes des dialogues; la chapelle diabolique de l'Epître dédicatoire à Dieu; le donjon de Vénus impudique entre ses trois poivrières : le projet des Maisons closes, le Traité de l'antiphysique et le Traité des goûts; (...) Telle se dressait, dans l'azur du meurtre et de la corruption érotique, la gigantesque Sodome de la Nature dévoilée » (Sade, Euvres complètes, édition de Gilbert Lely, Paris, Cercle du Livre Précieux, 1962, t. II, p. 607).

${ }^{3}$ Jean-Jacques Brochier, «La circularité de l'espace », dans Le marquis de Sade, Actes du Colloque d'Aix-en-Provence (1920 février 1968), Paris, Armand Colin, 1968, p. 171-184.
} 
étudiée. Ainsi, tandis que les quatre scélérats des Cent vingt journées s'emmurent volontairement dans leur château de Silling, «comme dans une citadelle assiégée, sans laisser la plus petite issue, soit à l'ennemi, soit au déserteur ${ }^{4} "$, les deux roués d'Aline et Valcour (Blamont, le père incestueux et son comparse Dolbourg qu'il veut unir à sa fille Aline) font au contraire profession d'assaillants d'une autre citadelle, le pucelage d'Aline : "Si tu ne t'y prends pas mieux que cela, cependant, s'emporte Blamont contre Dolbourg, je crains que nous ne soyons réduits à emporter la citadelle d'assaut. [...] Tant qu'une porte ne s'ouvre point à deux battants, tu n'imagines pas qu'il puisse y avoir des moyens de forcer les barricades ${ }^{5}$ »

La vertu d'Aline est symbolisée par les frontières de sa chambre, à l'étage de la maison maternelle, elle-même au cœur du domaine de Vertfeuille, considéré (à tort) comme un lieu protecteur. L'irruption brusque des deux complices de grand matin y prend des allures de siège : «quand il s'agit de recevoir un père et un époux, dit [Blamont] à travers la porte, on ne doit pas y regarder de si près : ouvrez, Aline, et n'ayez nulle crainte. » $(A V, 470)$.

Se dessinent ainsi, sur un même fond de métaphore militaire - poncif libertin par excellence - deux configurations symétriquement inverses : une place vertueuse que le libertin force; une place orgiaque que le libertin défend, fusse contre des assaillants imaginaires. De Vertfeuille à Silling (sites relativement contemporains dans la chronologie de l'œuvre de Sade), la citadelle change de camp. Et selon la perspective choisie, l'interprétation et les enjeux de ce déplacement varient. Si l'on passe du domaine assiégé de Vertfeuille au château muré de Silling, c'est un basculement qui s'impose. Basculement d'une topique traditionnelle du roman libertin (le roué forçant les résistances de ses victimes jusque dans leurs réduits intimes) vers un espace radical du fantasme (la citadelle sert d'écrin aux pulsions, dans une violence d'autant plus terrible qu'elle est confinée et claustrale). La retraite fragile des victimes de Vertfeuille sert alors d'étalon pour apprécier, dans l'autre versant de l'œuvre, la radicalité des forteresses étanches et effroyables qui n'ont presque plus rien des espaces traditionnels du roman noir. Inversement, si l'on chemine de Silling à Vertfeuille, on remarque non pas tant une atténuation de la violence, qu'une complexité nouvelle du lieu. La précarité de la citadelle vertueuse est certainement une faiblesse en termes militaires mais c'est aussi une force littéraire car en étant menacé et forcé le refuge gagne en ambiguïté : il reste (ou devient) un espace hétérogène et contradictoire, résultat de tensions et de résistances, où tous les glissements (du vice à la vertu, de l'amoenus au terribilis) sont possibles. L'enjeu est alors de restituer un peu de la subtilité de l'écriture " exotérique » de Sade, déjà mise en évidence par Michel Delon ${ }^{6}$, en nous appuyant cette fois-ci non plus sur le retraitement des intrigues ni sur le tremblement identitaire des personnages, mais sur la configuration des espaces.

\footnotetext{
${ }^{4}$ Sade, Les Cent vingt journées de Sodome, Euvres, édition de Michel Delon, Paris, «Bibliothèque de la Pléiade », t. I, p. 58. (Désormais abrégé en $C V J$ ). Nous soulignons.

${ }^{5}$ Sade, Aline et Valcour, Cuvres, édition citée, t. I, p. 418,(Abrégé en AV). Nous soulignons.

${ }^{6}$ Voir notamment l'introduction à son édition des Crimes de l'amour ( Paris, Gallimard, folio, 1987). Pour une mise au point récente sur la place de l'œuvre revendiquée de Sade (notamment les formes brèves du conte et de la nouvelle) et sur l'état de la critique, voir la présentation des Contes libertins de Sade dans l'édition qu'en a donnée Stéphanie Genand (Paris, Flammarion, GF, 2014).
} 
Notre idée est qu'il est possible de voir dans ces deux grandes configurations du retrait (le réduit fragile et «poreux »/ la forteresse impénétrable) les traductions spatiales emblématiques de deux modes d'écriture, c'est-à-dire de deux rapports différents au lecteur et aux sources. Rapport au lecteur d'abord, parce que la citadelle des fictions est à l'image de l'œuvre : c'est tantôt l'écrin secret où l'on peut tout dire, tout déplier et tout montrer, tantôt l'espace décent mais mêlé, où s'affrontent, non sans ironie, le vice et la vertu. Rapport aux sources ensuite, parce que le topos de la retraite, presque constitutif du roman des lumières ${ }^{7}$, est repris systématiquement par Sade, sous toutes ses formes, mais sur des modes fort différents selon les romans. Le même motif, simplement détourné par un jeu littéraire, dans un roman avoué, semble, dans le roman clandestin, littéralement arraché à la tradition littéraire, comme il est arraché au temps et à l'espace du récit pour ouvrir sur l'univers hors-temps du fantasme.

«Malgré la justice de la providence, le vice écrasera la vertu» $(A V, 1018)$. La plainte de Mme de Blamont, accablée par les complots de son mari, vaut comme un programme du roman, mais résume plus largement la poétique de l'œuvre exotérique de Sade, qu'il expose en des termes similaires dans son Idée sur les romans ${ }^{8}$. Si ce cri de désespoir rappelle les accents des lamentations de Justine, il s'en distingue pourtant par l'angoisse et l'incertitude exprimées par Mme de Blamont. Ces sentiments et pressentiments construisent l'idée d'une menace, d'une ombre qui plane au dessus des âmes sensibles. Sans même invoquer la trop fameuse formule de Foucault, «il n'y a pas d'ombre chez Sade », on sait que le mal dans Juliette ou les Justine s'abat mécaniquement sur la victime, dans une coïncidence absolue entre le projet du libertin et sa réalisation. Dans les nouvelles et romans «décents", au contraire, si on ne peut douter que le mal triomphera, on se donne au moins le temps de le redouter. Or, cette part laissée à l'angoisse et aux brouillards semble se traduire, dans l'espace, par des refuges instables, à la sécurité incertaine : des retraites toujours menacées d'invasion.

On a déjà mentionné Vertfeuille, l'îlot rousseauiste d'Aline et Valcour, qui emprunte au domaine de Clarens ses rythmes champêtres et ses âmes sensibles. Or, en détournant, en malmenant la société utopique des cœurs de la Nouvelle Héloïse (le rêve bucolique est caricaturé, ses hôtes sont faibles et crédules...) Sade en conserve au fond les contradictions fondamentales, et les déplie même au grand jour, comme le dit très justement Bérengère Durand ${ }^{9}$. Sade déplace en fait la contradiction toute intérieure de l'utopie de Clarens (le désir et son souvenir qui viennent miner le remède utopique de Wolmar) en lui substituant une menace plus franche (le désordre libertin), laquelle, par une minutieuse construction romanesque, surgit toujours de l'extérieur. La rencontre avec Sophie (jeune victime de Blamont, qu'on croira un temps sa fille) a lieu dans la

\footnotetext{
${ }_{8}^{7}$ Nous renvoyons ici aux nombreux travaux d'Hélène Cussac sur les topoï de la retraite dans le roman d'Ancien Régime.

${ }^{8}$ « Si, après les plus rudes épreuves, nous voyons enfin la vertu terrassée par le vice, indispensablement nos âmes se déchirent, et l'ouvrage nous ayant excessivement ému $[. .$.$] doit indubitablement produire l'intérêt, qui seul assure les$ lauriers. » (Idée sur les romans, dans Les Crimes de l'amour, ouvr. cité, p. 39.)

${ }^{9}$ L'Elysée «est une synthèse des contradictions qui tiraillent Julie et partant, le roman: il est une mise en espace problématique du refoulement de la passion de l'héroïne. Sade, en fin lecteur de Rousseau, retrouverait ironiquement, dans Aline et Valcour, ces contradictions qui minent la transparence de l'autarcie pastorale ». (Bérangère Durand, «Clarens à Vertfeuille : un tombeau pour l'Elysée », dans Sources et postérité de la Nouvelle Hélö̈se de Rousseau : le modèle de Julie, dir. Geneviève Goubier et Stéphane Lojkine, Paris, Desjonquères, 2012, p. 237.)
} 
forêt, lors d'une de ces «promenades éloignées », dont les «aventures » et les «dangers » avaient fait l'objet des inquiétudes ou des railleries ${ }^{10}$; quant à l'arrivée de Blamont et Dolbourg, ainsi que celle du couple Léonore-Sainville, elle fait l'objet d'une mise en scène dramatique suggérant l'irruption menaçante d'un élément étranger dans une harmonie fragile ${ }^{11}$. La vulnérabilité de la vertu, voire son «instabilité axiologique $»^{12}$ trouve ainsi leur traduction presque transparente - et ce n'est peut-être pas là la moindre des violences de l'écriture de Sade - dans l'espace : une retraite menacée et violée.

A l'autre extrémité de la carrière d' " homme de lettres » de Sade, dans La Marquise de Gange, son dernier ouvrage, le château où la vertueuse Euphrasie se retire avec son mari loin des perversités de la Cour, est un espace tout aussi incertain. L'intrigue s'ouvre sur cette retraite bruyamment préparée par les convictions champêtres de la jeune femme : «on ne s'aime jamais mieux qu'à la campagne $»^{13} ;$ « Eh bien ! Partons cher époux, quittons ce séjour dangereux de la galanterie et de la corruption; ce n'est pas où l'on parle toujours d'amour que je veux être, c'est où l'on sait mieux le sentir. Le château de tes pères me paraît si propre à remplir nos vues [...] On partit : l'antique et superbe château de Gange fut choisi pour le lieu de l'habitation » $(M G, 183)$. Écho des douces idées de Mme de Blamont ${ }^{14}$, ces paroles annoncent une retraite tout aussi précaire, mais sur un mode mélancolique et gothique. Le malheur qui menace est non seulement annoncé par une série de présages grossiers (une chute sur le chemin qui mène Euphrasie à Gange, suivie d'un mauvais rêve la nuit de l'arrivée), mais il est plus subtilement assumé par les contradictions de l'architecture même du lieu. A Gange, les efforts déployés pour aménager des appartements dans le confort moderne se heurtent à la vaste structure gothique de l'édifice. Ainsi, dans l'appartement d'Euphrasie, « le plus riche de la maison », « un meuble complet de soie vert et or [...] voilait ces pierres antiques élevées depuis près de huit siècles » $(M G, 189)$; et les «quelques cabinets » qui présentent «toutes les aisances", se détachent bien timidement sur fond d'architecture noire, à savoir « le reste de la maison, l'une des plus vaste de la province, (qui) répondait à ce style d'architecture et de distribution gothique, si précieux aux âmes sombres et mélancoliques » (Ibid.). Rien de tel, par exemple, dans le Château de Silling des Cent vingt journées, où le même type d'aménagements, au profit des libertins cette fois-ci, s'était réalisé sans aucun traitement dramatique ${ }^{15}$. Au contraire, les espaces

\footnotetext{
${ }^{10}$ Voir $A V$, Lettres dixième, onzième et quinzième.

11 «Hier au soir tout était calme... [...] Mme de Senneval, près d'une croisée donnant sur la cour, quitte son livre et dit effrayée : J'entends une voiture. » $(A V, 469) ;$ «M. de Beaulé jouait aux échecs avec Mme de Senneval ; il était environ 8 heures du soir, le ciel très obscur se remettait à peine d'un ouragan épouvantable, lorsque tout à coup nous entendîmes un homme à cheval faire retentir la cour de son fouet... de ses cris, et appeler à lui de toutes ses forces... » $(A V, 519)$

${ }^{12}$ Sur les profondes ambiguités du discours de la vertu dans Aline et Valcour, notamment les jeux de glissement et de contamination du discours sensible par les stratégies de séduction, voir l'article de Mladen Kozul «Subordinations et séductions épouvantables, les stratégies du dévoiement », dans Sade en toutes lettres, autour d'Aline et Valcour, dir. Catriona Seth et Michel Delon, Paris, Desjonquères, 2004, p. $44-59$ (ici p. 45); et celui de Michel Delon, «Le tremblement de l'identité », dans ouv. cité, p. 60-69.

${ }^{13}$ Sade, La Marquise de Gange, CEuvres Complètes du Marquis de Sade, Paris, Pauvert, 1991, t. XI, p. 182. (Abrégé en MG)

14 «C'est dans l'asile du repos et de l'innocence, c'est à la campagne, mon cher Valcour, où le bonheur de s'aimer se goûte mieux selon moi $\gg(A V$, p. 424).

${ }^{15}$ «Qu'on observe que je vais peindre les appartements non tels qu'ils pouvaient être autrefois, mais comme ils venaient d'être arrangés et distribués relativement au plan projeté. » $(C V J, 55)$
} 
hétérogènes du salon «commode et agréable » $(C V J, 55)$, du théâtre et du «cachot voûté » en souterrain $(C V J, 58)$, donnés tous ensemble comme d'un seul tenant, semblent parfaitement soudés par la puissance du fantasme. À Gange, le confortable et le terrible, bien que contigus (l'un des cabinets d'Euphrasie sert de « cage d'escalier » à la sombre tour des Archives) voire superposés (la soie vert et or qui «voile» les pierres antiques), ne sont mobilisés que successivement, à mesure que l'intrigue avance et que progressent les complots ourdis par le beau-frère d'Euphrasie, l'abbé Théodore de Gange. Le fragile équilibre est rompu pour la première fois par l'irruption de l'abbé :

\begin{abstract}
On venait de se rassembler dans le grand salon, qu'éclairait un lustre garni de bougies ; une partie d'hombre occupait M. et Mme de Gange, Mme de Roquefeuille, et le comte de Villefranche. Le Père Eusèbe, au coin de l'antique foyer de cette salle, éclaircissait un point de doctrine à Mlle de Roquefeuille. Six heures sonnaient au donjon du château, lorsqu'un grand bruit extérieur annonça l'arrivée d'un nouvel hôte. Les deux battants roulent avec fracas sur leurs gonds épais. $(M G, 196)$
\end{abstract}

Réécriture évidente des scènes d'irruption qui venaient troubler la petite société de Vertfeuille dans Aline et Valcour, le passage fait aussi entendre l'écho subtil des « deux battants » que Blamont incitait métaphoriquement Dolbourg à forcer. Par sa seule entrée fracassante, l'abbé de Gange signe son appartenance à la lignée des libertins les plus agressifs. Progressivement, ce château violé par Théodore va passer désormais sous son contrôle après l'éloignement du Marquis. L'espace se renverse alors en prison pour Euphrasie. Convaincue à tort d'adultère par l'abbé qui cherche à la perdre, elle est déportée dans un « réduit lugubre » de la tour des Archives, avec «barreaux de fer » et méchants meubles $(M G, 265)$. Une sortie nocturne pour rencontrer sa mère retenue dans l'aile opposée aggravera encore sa détention, lui valant le «mitard » souterrain, « dans le cachot de cette même tour où l'air pénétrait à peine » $(M G, 298)$. La retraite désirée des premiers épanchements (ces «retraites fleuries qu'il semble que la nature n'embellisse que pour l'amour » $(M G, 182)$ dégénère en retraite subie («dans la retraite où vous me condamnez » lance-t-elle un jour à l'abbé $(M G, 265)$.

Qu'il s'amuse à miner le locus amoenus de la retraite champêtre dans Aline et Valcour, ou qu'il joue, dans une veine noire, à renverser en carcieri le séjour vertueux de la demeure des pères, Sade reste au fond fidèle à une caractéristique essentielle du motif de la retraite dans les grands romans des Lumières, à savoir sa précarité. Quelles que soient les intrigues, la décision ou l'intimation de se retirer incarnent, dans la production romanesque, un point fragile et temporaire d'équilibre, qui participe à la fabrication du déséquilibre sans lequel il n'y a pas de récit ni d'histoire. On songe bien sûr à Prévost (que Sade plaçait si haut dans son Idée sur les romans ${ }^{16}$ ) dont les héros, toujours en quête d'un état chimérique de stabilité, pratiquent la retraite comme une solution récurrente, mais à jamais vaine. «Dans tous les romans de Prévost, expliquait fort bien Robert Mauzi, les retraites ne sont que des abris provisoires, constamment menacés et finalement inutiles [...] illusoires et fragiles dans un univers de la

\footnotetext{
${ }^{16}$ Idée sur les romans, ouv. cit., p. 40-41.
} 
Fatalité ${ }^{17}$. Les retraites des écrits « avouables » de Sade gardent bien quelque chose de ce déséquilibre, de ces ambiguïtés propres aux refuges du roman des Lumières. Parce que la tranquillité du vertueux y est toujours dérangée, bien sûr, mais aussi, inversement, parce que le crime s'y teinte parfois d'honnêteté. C'est le cas du repaire du bandit Franlo dans Faxelange, l'histoire qui ouvre les Crimes de l'amour. Le brigand Tranche-Montagne, usurpant l'identité d'un Baron de Franlo, obtient la main de Mlle de Faxelange. Séduite par sa belle position, elle croit le suivre dans ses terres des Amériques. Dévoilant bien vite son vrai visage, le brigand entraîne sa femme effondrée dans son repaire cévenol, « retraite [...] introuvable ${ }^{18}$ où elle endossera, bon an mal an, le rôle de la femme du chef. Elle sera finalement délivrée par une troupe de dragons commandée par Goé, l'amant de toujours qu'elle avait délaissé pour le mirage Franlo. Repaire de brigands dans la tradition du roman picaresque, le site possède un statut moins tranché que le nom de son capitaine. Certes, la «petite plaine », qualifiée par Goé de «local $[\ldots]$ horrible » $(C A, 88)$ est presque aussi impénétrable que celle de Silling, dont Sade semble raviver le souvenir sous une plume décente : elle est « resserrée de toutes parts par des montagnes inaccessibles » $(C A, 79)$ comme celle de la forêt noire était «entourée de partout de rochers à pic » $(C V J, 55)$; «on ne pouvait (y) pénétrer que par (un) seul sentier » là où le chemin du pont, à Silling «était l'unique qui puisse descendre et communiquer dans la petite plaine »; enfin, le «poste de dix de ces scélérats [...] qui veillaient constamment jour et nuit » à l'entrée des gorges rappelle le village «de voleurs et de contrebandiers » $(C V J, 54)$ qui sert de première redoute à Silling. Mais, loin d'enceindre le vice tout pur pour le protéger, le hameau de TrancheMontagne mêle le crime et l'honnêteté par le biais du pacte, si ce n'est d'amour, du moins de confiance, qui se noue entre les époux. Franlo confie à sa vertueuse femme les tâches les plus cruciales et cruelles (dépouiller les victimes et les faire égorger); inversement Faxelange, abandonnant la plainte pour la «fermeté », finit pas endosser son rôle de mère Brigand. La relativisation du vice dans le repaire de forbans n'est pas chose nouvelle dans le roman. Là encore, Sade peut jouer d'une tradition picaresque qui avait déjà associé le séjour chez les voleurs à une expérience mitigée. Gil Blas, par exemple, éprouve des sentiments mêlés au fond du souterrain où le retiennent les bandits. S'il feint la gaieté par stratégie, il est tout de même spectateur d'une existence joyeuse et agréable ${ }^{19}$. Mais ce qu'il y a de remarquable avec le repaire de TrancheMontagne, c'est que cette ambiguïté est marquée spatialement, voire architecturalement :

\footnotetext{
Une fois dans la plaine, on trouvait une mauvaise bourgade, formée d'une centaine de huttes, à la manière des sauvages, à la tête desquelles était une maison assez propre, composée de deux étages, partout environnée de hauts murs et appartenant au chef. C'était là son séjour, et en même temps la citadelle de la place, l'endroit où se tenaient les magasins, les armes et les prisonniers ; deux souterrains profonds et bien voûtés servaient à ces usages ; sur eux, étaient bâtis trois petites pièces au rez-de-chaussée, une cuisine, une chambre, une petite salle, et audessus un appartement assez commode pour la femme du capitaine. $(C A, 78)$
}

\footnotetext{
${ }^{17}$ Robert Mauzi, «Le thème de la retraite dans les romans de Prévost », dans L'Abbé Prévost, Actes du Colloque d'Aix, Ophrys, 1965, p. 195.

${ }^{18}$ Sade, Faxelange ou les torts de l'ambition, dans Les crimes de l'amour, ouvr. cité, p. 86 (abrégé en CA).

${ }^{19}$ Voir Lesage, Histoire de Gil Blas de Santillane, Paris, Flammarion, « GF », 1977, p. 39.)
} 
Une nette opposition divise tant dans le plan du domaine, entre les huttes et l'honnête maison, que dans l'élévation de la maison, entre les locaux techniques des brigands au sous-sol et les quartiers de Madame à l'étage. La maison oscille entre forteresse du crime et respectable résidence. La manière dont notre couple a priori si mal assorti va consommer cet espace vient confirmer cette confusion morale. Le jour où Goé surgit avec sa troupe pour délivrer Faxelange, elle est occupée à jouir paisiblement des délices de sa propriété : «on était au mois d'octobre, Franlo et sa femme dînaient ensemble sous une treille, à la porte de leur maison, lorsque dans l'instant dix ou douze coups de fusil se font entendre au poste» $(C A, 87)$. La posture des époux rappellerait presque celle de saints ermites des romans de Loaisel de Tréogate ${ }^{20}$. Quant à l'effraction par les dragons de Goé d'un local réputé « introuvable » et «impénétrable », elle fait écho aux scènes d'irruption menaçante au cœur des veillées vertueuses d'Aline et Valcour. Elle semble en inverser le sens (puisque la loi et la vertu vont triompher militairement du brigandage et de la séquestration), mais ajoute en réalité à la confusion car la violence et l'agression sont ici du côté de Goé. Le « poste » attaqué est situé, notons-le, «à la gorge du sentier $\gg(C A, 78)$. Quoi qu'il en soit, ici comme ailleurs, le viol d'une retraite est la conséquence de sa fragilité interne : porosité au vice pour la retraite sensible ; porosité à la vertu pour la retraite du brigand.

Ce sont les retraites de l'autre versant de l'œuvre qui sont les mieux connues. Leur étrangeté fascine, et de toute façon on ne saurait les manquer tant elles saturent le récit. La fiction tout entière des Cent vingt journées semble s'engloutir dans le château qu'elle déploie, et Justine et Juliette, pour peu qu'on épure leurs aventures, suivent un trajet qui passe de retraite en retraite comme par autant de stations. Dans la seule Histoire de Juliette, on trouve le couvent et le caveau (Delbène), les petites maisons secrètes et confortables (La Duvergier, Dorval et la Durand) parfois doublées de jardins agréables (Olympe Borghèse), les retraites urbaines (la Société des Amis du Crime), ou agrestes (les citadelles de Minski ou Crodelli), les réduits sauvages (l'̂̂le déserte de Brisa-Testa) ou primitifs (la hutte sibérienne du même). La nouvelle Justine qui ajoute le repaire escarpé (Roland) ou l'auberge forestière (M. d'Esterval), présente un peu moins de variété dans les modèles, mais leur enchaînement y est plus systématique et beaucoup mieux articulé, du fait que l'héroïne est une séquestrée en puissance. Il arrive ainsi que la liaison entre les épisodes se fasse par le biais d'une surenchère dans la sévérité des retraites ${ }^{21}$.

Sade recycle en virtuose l'éventail exhaustif des retraites topiques du roman des Lumières, mais c'est pour en confisquer brutalement les significations. Les raisons de se

\footnotetext{
${ }^{20}$ Ainsi le Comte de la Gleh dans Ainsi finissent les grandes passions : «Tout était riant et ouvert dans cette paisible retraite ; tout y annonçait la confiance et la sécurité. Le Comte De La Gleh prenait le frais sous un berceau devant sa maison. Il était assis tranquillement à une petite table, l'air doux et recueilli, et paraissait en ce moment occupé d'idées beaucoup moins terrestres que celles dont alors mon âme était la proie. » (Loaisel de Treogate, Ainsi finissent les grandes passions, ou les dernières amours $d u$ Chevalier de..., Lettre 42, p. 21.)

${ }^{21}$ Par exemple entre le château de Bandole et le couvent de Sainte-Marie-des-Bois. Le château «a l'air du monde le plus sombre et le plus sauvage. »; "Si Justine avait cru les abords du château de Bandole d'un agreste effrayant, certes elle dut trouver ceux de cette abbaye bien plus sauvages encore. »(Sade, La Nouvelle Justine, Euvres, Paris, Gallimard, Bibliothèque de la pléiade, t. II, p. 573 et 592). (Abrégé en NJ).
} 
retirer, qui sont multiples dans les intrigues des romanciers ${ }^{22}$, il les ramène à une seule et unique tout au long de son œuvre : l'assouvissement des pulsions. Aspiré par la force du fantasme, le motif du retrait, qui faisait l'objet de réécritures et de détournements dans l'œuvre décente où il jouait encore un rôle diégétique, est ici littéralement arraché à la tradition romanesque. Entièrement creusées par le fantasme, les claustrations du libertin s'affranchissent de toute autre nécessité que celle de la jouissance - à commencer par les nécessités de l'intrigue. La retraite fortifiée ne répond à aucun impératif extérieur, à aucun besoin réel de fuir et de se cacher, elle n'est la solution d'aucun problème. C'est Minski qui formule cet étrange paradoxe : retranché dans sa forteresse, derrière les rideaux de montagnes, de forêts, d'eau, de murailles et de terre, le géant avoue : «ce n'est pas que j'aie la moindre chose à craindre ; ceci tient aux Etats du grand duc de Toscane, on y connaît toute l'irrégularité de ma conduite et l'argent que j'y sème, me met à l'abri de tout ${ }^{23}$. Protégé d'abord par la plus solide des redoutes, l'argent, le libertin, au reste parfaitement célèbre, ne se retire que par plaisir (Minski parle d'ailleurs des « plaisirs de [sa] retraite » qu'il tient à faire partager à ses visiteurs) (HJ, 701). Dès lors les refuges variés du libertinage ne seraient-ils pas, à l'échelle de l'ensemble de l'œuvre, comme « six-cent lieux divers » qui accompagneraient « les sixcent plats divers » annoncés en préambule des Cent-vingt journées (CVJ, 69), autrement dit des compléments circonstanciels de la jouissance, ajoutant au plaisir de chacune des passions celui du cadre où elle s'exerce ? C'est possible, bien qu'aucun libertin ne théorise la chose aussi nettement. Toujours est-il qu'à l'instar des six cent passions racontées par les historiennes de Silling, la seule évocation d'une retraite suffit à provoquer la jouissance : «quand on fit la description [du caveau] au duc, il en déchargea trois fois de suite» $(C V J, 58))$. Arrêter sa pensée sur le local où l'on se trouve suffit à échauffer l'imagination : à Sainte-Marie-des-Bois, Omphale précise que «rien n'embrase [les moines], rien ne leur monte l'imagination, comme l'impunité que leur promet cette inabordable retraite » $(N J, 640)$. Il y a là comme une inscription dans la fiction du plaisir de l'écrivain Sade à forger des lieux imaginaires, indépendamment de toute intrigue. Car la clôture terrible de l'abbaye, on le sait, ce sont les moines qui l'inventent et l'imaginent en partie - et après eux leurs victimes. En effet, cette «septième enceinte » imaginaire $(N J, 640)$ qui s'ajouterait aux six haies vives qui entourent le pavillon, cette «épaisse muraille» que ne trouvera jamais Justine lors de son évasion, c'est la jeune Omphale, codétenue de Justine, qui la lui dépeint. A aucun moment les moines n'en parlent directement. Dans sa harangue d'accueil, Sévérino, le moine supérieur, s'en tient à « six enceintes de dix pieds chacune » $(N J, 608)$. Et c'est une remarque a posteriori du narrateur (que l'on est tenté de prêter à Justine) qui évoque une allusion par les prêtres, que le lecteur n'a pas entendue («il est vraisemblable que les moines ne le disaient que pour effrayer davantage » $(N J, 816)$. Cette circulation, plus ou moins confuse, du discours sur la muraille imaginaire

\footnotetext{
${ }^{22}$ Voir Hélène Cussac, «Les lieux de retraite dans quelques romans du $18^{\mathrm{e}}$ siècle : d'une géographie réelle à la remise en cause d'un espace utopique », dans Geographiae imaginariae. Dresser le cadastre des mondes inconnus dans la fiction narrative de l'Ancien Régime, éd. Marie-Christine Pioffet, Presses Universitaires de Laval, Québec, 2011, p. 139-161.)

${ }^{23}$ Sade, Histoire de Juliette, Pléiade, éd. cit., t. 3, p. 704. (Abrégé en HJ).
} 
contribue à la déréaliser encore un peu plus, en suggérant qu'elle relève de la création de la fiction elle-même.

Toute pétrie d'imaginaire, la retraite du libertin est dépourvue des contradictions qui caractérisaient la retraite romanesque. Elle est certainement «négative » au sens où elle est tendue vers la destruction et la mort. Mais elle est totalement exempte de cette «négativité » que Jean Sgard attribue aux retraites du roman de Prévost, lieux de «déroute, de défaite ${ }^{24}$, fragilisés par cette tension, véritable dynamique du roman, entre les valeurs idéales associées au refuge et les contraintes du monde extérieur. Cette négativité, on a vu que le Sade homme de Lettres en assumait l'héritage, qu'il la récupérait, avec le legs du roman sensible ou noir, pour la transposer. Le Sade pornographe en revanche efface cette négativité et lui substitue la positivité presque magique d'un ordre qui s'applique immédiatement et sans obstacle. Là où il fallait à Cleveland plusieurs pages et sans doute des jours de réflexions tourmentées pour déterminer la forme et le lieu de sa retraite à Saumur, les libertins de Sade héritent comme par magie d'un site qui sied parfaitement à leurs projets, sans qu'ils aient besoin de s'en justifier longtemps : «c'est ce qui me convient » répond laconiquement Roland à Justine qui s'étonne du site plutôt escarpé de son repaire $(N J, 1009)$. Ils s'y retirent tout naturellement ${ }^{25}$, comme si l'endroit enveloppait et épousait leur existence.

Dépouillée de tout enjeu véritablement diégétique, roc immobile, poids mort du romanesque d'intrigues, la retraite, à l'image des souterrains qu'elle dissimule bien souvent, exprime des vérités profondes et fondamentales. Elle peut se lire tout à fois comme une allégorie de l'économie pulsionnelle et une traduction de «l'isolisme» philosophique des libertins. Considérons par exemple la hutte primitive de Brisa-Testa dans son exil sibérien. Fait extrêmement rare dans le roman sadien, ce misérable refuge est imposé au libertin qui ne le choisit pas pour son plaisir. Et pourtant, sous les couches de références anthropologique (la précision des matériaux de construction) et philosophique (l'état de nature anti-rousseauiste), affleure la signification profonde de la masure : comme un décor monté de toutes pièces par le fantasme, elle exprime l'isolement agressif de l'individu et la cruauté des pulsions.
Sa construction était en sapin parqueté d'os de poisson, qui rendaient le plancher aussi luisant que l'ivoire; il y avait au-dessus un bouquet d'arbres assez pittoresque ; et pour se mettre à l'abri de l'incursion de bêtes sauvages, on y avait creusé un fossé palissadé avec de forts poteaux et des pièces de bois en travers; cette barricade était armée de pointes qui formaient comme autant de lances ; et lorsque les portes étaient fermées, on y était aussi en sûreté que dans une place forte; $[\ldots]$ je passai près de dix ans dans cette cruelle retraite, n'ayant d'autre société que quelque infortunés comme moi. $(J, 980)$

Comme au château de Silling, où l'assaillant n'est, selon l'euphémisme, " pas même à redouter », de bêtes sauvages il ne sera jamais question ici - sauf à les chercher à l'intérieur de la hutte. Les pointes et les lances ne peuvent qu'être métonymiques

\footnotetext{
${ }^{24}$ Jean Sgard, «La vie paisible et solitaire selon Prévost », dans Les loisirs et l'héritage de la culture classique, Actes du $13^{\mathrm{e}}$ Congrès G. Budé, Latomus, n 230 (1996), p. 615.

${ }^{25}$ «Retiré du monde dès l'époque où il avait hérité de son père, Bandole, depuis plus de quinze ans, se livrait, dans cette solitaire habitation, aux goûts bizarres qu'il avait reçus de la nature » $(N J, 572)$
} 
d'autres armes et d'autres tortures, car la retraite est profondément centripète. Portes fermées, les libertins n'ont plus rien à faire avec le monde extérieur, pas même à s'en défendre. Parce que l'extérieur a tout simplement disparu. Il est remarquable que l'effraction ou même sa tentative, scénario récurrent du roman décent, soit totalement absente des romans pornographiques. A l'exception d'un cas de règlement de compte entre libertins ${ }^{26}$, les seuls refuges violés sont précisément ceux qui abritent les vertueux : le château de l'honnête famille de Cloris, où sont retenues un temps Juliette et Clairwill par un père qui réclame justice pour son fils, sera très rapidement pris d'assaut par les troupes de Saint-Fond ${ }^{27}$; dans l'affreux repaire des faux-monnayeurs de La Nouvelle Justine, le remplacement de Roland par Delville, brigand plus honnête, suffit à fragiliser la place. Elle est bientôt libérée par les forces de l'ordre. «Le malheureux Delville était honnête dans sa profession ; n'en était-ce pas plus qu'il n'en fallait pour être promptement écrasé ? [...] les portes s'enfoncent tout à coup, les fossés s'escaladent, et le château, avant que ceux qui l'habitent aient le temps de songer à leur défense, se trouve rempli de plus de soixante cavaliers de maréchaussée » $(N J, 1039)$. Comme une bactérie qui fragilise un organisme, une once d'honnêteté inoculée dans la forteresse finit par la ruiner. C'est la preuve par l'architecture ou par la fortification, des infortunes de la vertu.

Retraites fragiles ou retraites impénétrables; retraites forcées de l'extérieur ou citadelles sans extérieur : ces types de décor ne sont pas des motifs différents au sein du paysage romanesque sadien. Recoupant exactement les frontières entre l'œuvre exotérique et l'œuvre ésotérique, elles sont des marqueurs de stratégies d'écriture radicalement opposées. L'une qui fonctionne par réécriture, détournement de topoï du roman des Lumières pour y glisser, dans les interstices, une dose de perversion qui les mine $^{28}$. L'autre qui, comme ces forteresses dans des déserts sans ennemis, rompt littéralement des amarres avec les traditions littéraires en vidant les lieux de leur ancienne substance romanesque pour n'y faire plus résonner que le bruit des fantasmes.

\section{Fabrice Moulin Université Paris Ouest Nanterre / CSLF}

\footnotetext{
${ }^{26}$ Cœur-de-Fer obtient de Justine, prisonnière de Bandole, de pénétrer dans le château pour y enlever une femme dont il veut se venger : "vous nous montrerez la porte de cette maison magique » $(N J, 588)$ - Justine sera libérée à cette condition.

${ }^{27}$ Juliette et Clairwill sont enlevées et menées «dans un château fort solitaire [...] les portes se ferment ; nous entendons même qu'on les barricade en dedans » $(H J, 489)$. Prévenu par une ruse de Juliette, Saint-Fond envoie ses troupes qui « assiège[nt] le château » « forçant notre chambre » (HJ, 493 et 496).

${ }^{28}$ Dans l'introduction de son Sade, une esthétique de la duplicité (Paris, Classiques Garnier, 2014), Chiara Gambacorti choisit précisément, pour classer les deux pans de l'œuvre de Sade (classification dont elle montre d'ailleurs très finement la difficulté), le critère de la présence, dans les œuvres dites décentes, d'un double discours, qui vient vicier, subvertir, le pacte de lecture affiché. Cette duplicité suppose nécessairement un jeu avec les conventions et topoï romanesques, que l'œuvre pornographique n'a pas à jouer.
} 